\title{
Olanzapine Use for the Adjunctive Treatment of Adolescents with Anorexia Nervosa
}

\author{
Mark L. Norris, M.D., ${ }^{1-3}$ Wendy Spettigue, M.D., ${ }^{2-4}$ Annick Buchholz, Ph.D., ${ }^{2,3,5}$ Katherine A. Henderson, Ph.D., ${ }^{2,3,5}$ \\ Rebecca Gomez, B.Sc., ${ }^{2,3}$ Danijela Maras, B.Sc., ${ }^{3,6}$ Isabelle Gaboury, Ph.D., ${ }^{3}$ and Andy Ni, M.Sc. ${ }^{3}$
}

\begin{abstract}
Objective: To examine assessment and treatment profiles of adolescent patients with anorexia nervosa and eating disorder not otherwise specified who received olanzapine as compared with an untreated matched sample.

Method: A retrospective, matched-groups comparison study was completed. Medical files of 86 female patients treated in the eating disorder program at the Children's Hospital of Eastern Ontario were examined. Patients treated with olanzapine were initially identified through chart review and then matched to a diagnosis, age, and, when possible, treatment group that served as the active comparator. Weight gain was examined in a sample of 22 inpatients.

Results: Patients treated with olanzapine displayed greater evidence of psychopathology and medical compromise at the time of first assessment compared with those not treated. Rate of weight gain was not statistically different between groups when olanzapine was started during inpatient admissions. Medication effect on eating disorder cognitions could not be assessed given the presence of multiple confounders relating to treatment. Notable side effects included sedation and dyslipidemia in $56 \%$ of patients.

Conclusions: Despite our best attempts at matching olanzapine-treated subjects with a control sample, analysis revealed significant differences between groups, suggesting greater illness severity in those augmented with olanzapine. Given these inherent differences, we were unable to draw any firm conclusions regarding the potential efficacy of olanzapine. Factors associated with the prescription of adjunctive pharmacotherapy in this cohort appear to be linked to illness severity, acuity, and associated comorbidity. The observed side-effect profile indicates the need for more consistent predrug screening and for closer monitoring during treatment.
\end{abstract}

\section{Introduction}

A NOREXIA NERVOSA (AN) is a complex illness characterized by destructive weight loss behaviors that result from an interplay of debilitating cognitive, emotional, and physical processes (American Psychiatric Association 2006). AN often develops in adolescence and is found to be most prevalent in youth, with the peak age of onset between 15 and 19 years (Lucas et al. 1991).

The current treatment philosophies of many programs, including our own, draw on the principles of the Maudsley model of familybased treatment for AN. This approach encourages parents to take control of their child's illness while at the same time externalizing the eating disorder. Using an outpatient setting, parents are supported through the refeeding process using regular family therapy along with close medical follow-up and when needed, dietary counseling. In certain cases (i.e., medical compromise, medical deterioration during treatment, or failure of outpatient treatment), more intensive treatment is undertaken within the hospital using either a specialized inpatient or partial hospitalization (day treatment) program. Optimized treatment in these settings utilizes a multidisciplinary approach that draws on medical and nutritional rehabilitation as well as psychological interventions that involve individual, family, and group therapy (Garner and Garfinkel 1997).

To date, pharmacological treatments have not been shown to significantly affect outcomes during the acute phase of illness (Rivas-Vazquez et al. 2003). "Atypical" antipsychotic medications such as olanzapine have shown some promise as a possible adjunctive treatment option for patients with $\mathrm{AN}$, as evidenced by improved weight restoration, decreased levels of anxiety, and ruminating thoughts involving food and body image. However, large

\footnotetext{
${ }^{1}$ Department of Paediatrics, Children's Hospital of Eastern Ontario, University of Ottawa, Ottawa, Ontario, Canada.

${ }^{2}$ Children's Hospital of Eastern Ontario Regional Eating Disorder Program, Ottawa, Ontario, Canada.

${ }^{3}$ Children's Hospital of Eastern Ontario Research Institute, Ottawa, Ontario, Canada.

${ }^{4}$ Department of Psychiatry, Children's Hospital of Eastern Ontario, University of Ottawa, Ottawa, Ontario, Canada.

${ }^{5}$ Department of Psychology, Carleton University, Ottawa, Ontario, Canada.

${ }^{6}$ Department of Psychology, University of Ottawa, Ontario, Canada.

Funding and Support: Financial assistance was generously provided by the W Garfield Weston Foundation, Toronto, Ontario, Canada.
} 
randomized studies are lacking (Boachie et al. 2003; Barbarich et al. 2004; Mondraty et al. 2005; Brambilla et al. 2007; Mehler-Wex et al. 2008). A recent double-blind placebo-controlled trial in an adult population provided evidence for efficacy in patients treated with olanzapine as indicated by greater rates of weight gain, earlier achievement of target body mass index (BMI), and a greater rate of decrease in obsessive symptoms (Bissada et al. 2008). Given the lack of replicated large-scale studies, olanzapine use is still considered "off label" for the augmented treatment of AN and should be used with caution. Olanzapine is a thienobenzodiazepine whose mechanism of action is assumed to lie in its ability to block dopaminergic and serotonergic receptors (D1-4 antagonism and 5HT $2 \mathrm{~A} / 2 \mathrm{C}$ antagonism) (Eli Lilly and Company 2008). It is indicated for the acute and maintenance treatment of schizophrenia and other psychotic disorders, as well as for the acute treatment of manic or mixed episodes in bipolar I disorder (Eli Lilly and Company 2008). Since the number of studies examining the drug's efficacy has increased, so too has the recognition that olanzapine use is associated with an unfavorable side-effect profile, including metabolic disturbances, diabetes mellitus (DM), and atherogenic lipid profiles (Citrome 2007; Tschoner et al. 2007). To date, there have been no major outcome data that have reported on whether olanzapine has the propensity to result in metabolic disturbances in low-weight patients with AN.

The primary objective of this study was to compare assessment profiles (i.e., medical measures such as BMI, scores on psychological profiles, diagnosed comorbidity, etc.) as well as treatment course (i.e., intensity of treatment required, number of admissions, total treatment length, etc.) of a sample of adolescent women in whom AN or eating disorder not otherwise specified (EDNOS, anorexia spectrum) was diagnosed and who were treated with olanzapine, with a diagnosis, age, and, when possible, treatmentmatched cohort that did not receive olanzapine or any other antipsychotic. The secondary objective was to investigate the prevalence of medical and metabolic side effects encountered during olanzapine treatment. It was hypothesized that the two groups would differ in their psychological profiles at baseline in that the olanzapine-treated group would present with greater psychopathology when compared with those not adjunctively treated with olanzapine.

\section{Method}

A retrospective cohort study was completed. Adolescent women between the ages of 10 and 17 years in whom Diagnostic and Statistical Manual of Mental Disorders, 4th edition (DSM-IV) (American Psychiatric Association 1994) AN (either restricting or binge/purge type) or EDNOS (anorexia spectrum, BMI $<17.5$ ) was diagnosed between January 1, 2000 and December 31, 2006 at our hospital and who were treated with olanzapine were included in the analysis. Patients treated with olanzapine were initially identified by a chart review, and a matched cohort was identified thereafter. Patients treated with olanzapine were matched to control subjects initially by age and diagnosis at assessment, and then treatment intensity level was undertaken after assessment (i.e., outpatient care, entry into our day program, or admission to the inpatient unit). In an attempt to limit confounding variables but still examine the potential effect of medication on weight gain, a secondary analysis involving a cohort of patients admitted after first assessment and initiated on olanzapine for a minimum of 2 weeks and within 2 weeks of their assessment was also completed. Patients with a concurrent diagnosis of psychosis or a concurrent illness with psychotic features, or whose primary treatment was not under the direction of our eating disorder (ED) team, were excluded from the study.

Descriptive data were collected, which examined demographic information, diagnosis, and clinical characteristics provided at first assessment, including scores on the Children's Depression Inventory (Kovacs 1981), Multidimensional Anxiety Scale for Children (March 1997), and the Eating Disorder Inventory-2 (Garner 1991) Drive for Thinness and Body Dissatisfaction subscales. Screening laboratory data collected over the course of treatment, as well as neuro-imaging reports, were also examined. We defined screening laboratory tests as baseline blood work (fasting glucose, fasting lipid profile including cholesterol, high density lipoprotein (LP), and low density LP, liver function testing, hemoglobin A1c) completed within 1 month of olanzapine initiation and baseline electrocardiogram (ECG) completed within 2 months of olanzapine initiation. Information pertaining to number of hospitalizations, length of treatment, clinical indicators of progress such as weight gain, and medication-specific variables were collected and compared between groups. A detailed review of clinical notes was also undertaken in an attempt to categorize and define any noted problems or issues relating to the tolerability and side-effect profile of olanzapine.

\section{Statistical analysis}

Descriptive statistics were used to present the sample profile using mean, standard deviation (SD), median, interquartile range, and percent. Where appropriate, 95\% confidence intervals (CI) were calculated using the Wilson score method (Newcombe 1998). The Student's $t$-test and McNemar's chi-square tests of independence were performed using the statistical package SPSS 16.0. A two-tailed alpha level of 0.05 was used for all statistical analyses. Due to the retrospective nature of the study, informed consent was not required. The Research Ethics Committee at our hospital approved the study.

\section{Results}

Forty-seven out of 328 patients (14\%) assessed and/or treated for an $\mathrm{ED}$ at our center received olanzapine during the study timeframe. Of those treated, four patients were excluded, as they did not meet full inclusion criteria (one male patient, three patients received treatment outside our team while on medication). Of the 43 patients who remained, the restricting subtype of AN was the most common diagnosis provided at assessment (Table 1). Almost all patients treated with olanzapine received a comorbid psychiatric diagnosis at the time of assessment, of which anxiety disorders and depression were the most common. These rates were significantly higher than those witnessed in the comparison sample (Table 2). No

Table 1. Frequency and Percentage of Eating Disorder Diagnoses as Determined at Initial Assessment in a Sample of 43 Matched Pairs of Participants

\begin{tabular}{lc}
\hline Eating disorder diagnosis & Frequency $(\%)$ \\
\hline ANR & $29(67.4)$ \\
ANBP & $2(4.7)$ \\
EDNOS-R & $12(27.9)$ \\
Total & $43(100)$ \\
\hline
\end{tabular}

$\mathrm{ANR}=$ Anorexia nervosa restricting subtype; $\mathrm{ANBP}=$ Anorexia nervosa binge-purge subtype; EDNOS-R = Eating disorder not otherwise specified restricting subtype. 
Table 2. Frequency and Percentage of Patients Treated with Olanzapine $(N=43)$ with Comorbid Psychiatric Diagnoses of Anxiety, Depression, and Obsessive Compulsive Disorder Versus Patients Not Treated with Olanzapine $(N=43)$

\begin{tabular}{lccc}
\hline Comorbid psychiatric diagnoses & $\begin{array}{c}\text { Olanzapine Frequency }(\%) \\
(\mathrm{N}=43)\end{array}$ & $\begin{array}{c}\text { Comparison Frequency }(\%) \\
(\mathrm{N}=43)\end{array}$ & $\mathrm{p} \mathrm{Value}$ \\
\hline Anxiety & $29(67)$ & $13(30)$ & $<0.0001$ \\
Depression & $26(60)$ & $15(35)$ & 0.043 \\
Obsessive compulsive disorder & $3(7)$ & $1(2)$ & 0.625 \\
\hline
\end{tabular}

${ }^{\mathrm{a}} \mathrm{McNemar}$ chi-square test for paired data; binomial distribution used.

differences were found in selective serotonin reuptake inhibitors use at assessment between matched groups in whom a mood and/or anxiety disorders were diagnosed (25 pairs, $p=0.63)$.

Information relating to age, length of symptoms predating assessment (chronicity), BMI, and scores on psychological measures has been presented in Table 3. Although age at assessment was significantly different between groups, this difference is not clinically significant. As noted, patients treated with olanzapine presented at lower overall weights than their matched controls. Although analyses indicate that the olanzapine group scored higher than the comparison group on all evaluated psychological measures completed at assessment, none of the scores differed significantly. All other markers of illness reported at assessment were similar between groups.

\section{Analysis of weight gain}

In order to limit potentially confounding effects relating to treatment status, nutritional intake, and so on, a secondary analysis of inpatients who received olanzapine for a minimum of 2 weeks and within 2 weeks of their first ED admission were analyzed along with the appropriate diagnosis, age, and treatment-matched group $(N=11$ pairs). Although mean $t$-scores of depression remained higher in the olanzapine-treated inpatient subgroup, differences in anxiety scores were no longer significant (Table 4). Moreover, scores on other psychological measures completed at assessment remained slightly higher in the olanzapine-treated group (although not significant). BMIs between groups at admission were equal, although they were greater in the olanzapine-treated group at the time of discharge as a consequence of length of stays that were significantly longer. Rate of weekly weight gain was also calculated and although it was found to be higher in the olanzapinetreated group, the result did not reach statistical significance. Observed rates of readmission for ED treatment over the next year were greater for those treated with olanzapine, despite having higher BMIs at the time of discharge and equal proportions of patients transitioning into the day-treatment program postdischarge.

Table 3. Mean Medical and Psychological Variables of Interest at the Time of Initial Assessment, and Treatment Outcome Variables for Participants Who Were Adjunctively Treated with Olanzapine Versus Those Who Were Not Adjunctively Treated with Olanzapine

\begin{tabular}{|c|c|c|c|c|}
\hline Baseline characteristics & $\begin{array}{l}\text { Olanzapine Mean }(S D) \\
\quad(\mathrm{N}=43)\end{array}$ & $\begin{array}{l}\text { Comparison Mean (SD) } \\
\qquad(\mathrm{N}=43)\end{array}$ & $\mathrm{t}(d f)$ & $\mathrm{p}$ Value $^{\mathrm{a}}$ \\
\hline Age at assessment (years) & $14.4(1.9)$ & $14.8(1.6)$ & $-2.916(42)$ & 0.006 \\
\hline Disease chronicity (months) & $10.63(6.9)$ & $11.56(8.5)$ & $-0.587(42)$ & 0.560 \\
\hline BMI at assessment $\left(\mathrm{kg} / \mathrm{m}^{2}\right)$ & $15.9(1.9)$ & $17.0(2.8)$ & $-2.606(42)$ & 0.010 \\
\hline $\begin{array}{l}\text { Baseline psychological measures } \\
\text { relating to comorbidity }\end{array}$ & $\begin{array}{l}\text { Olanzapine Mean }(S D) \\
\qquad(\mathrm{N}=30)\end{array}$ & $\begin{array}{l}\text { Comparison Mean }(S D) \\
\qquad(\mathrm{N}=30)\end{array}$ & $\mathrm{t}(d f)$ & p Value ${ }^{\mathrm{a}}$ \\
\hline CDI t-score & $63.3(17.3)$ & $57.2(15.5)$ & $1.242(29)$ & 0.218 \\
\hline MASC t-score & $58.3(11.4)$ & $55.1(10.3)$ & $1.156(29)$ & 0.268 \\
\hline $\begin{array}{l}\text { Baseline ED-specific psychological } \\
\text { measures }\end{array}$ & $\begin{array}{l}\text { Olanzapine Mean }(S D) \\
\quad(\mathrm{N}=26)\end{array}$ & $\begin{array}{l}\text { Comparison Mean (SD) } \\
\qquad(\mathrm{N}=26)\end{array}$ & $\mathrm{t}(d f)$ & $\mathrm{p}$ Value $^{\mathrm{a}}$ \\
\hline Drive For Thinness ${ }^{\mathrm{b}}$ & $14.0(7.6)$ & $11.5(7.1)$ & $1.186(25)$ & 0.247 \\
\hline Body Dissatisfaction $^{b}$ & $14.2(9.8)$ & $11.4(8.3)$ & $1.026(25)$ & 0.315 \\
\hline Treatment course & $\begin{array}{l}\text { Olanzapine Mean }(S D) \\
\qquad(\mathrm{N}=43)\end{array}$ & $\begin{array}{l}\text { Comparison Mean (SD) } \\
\qquad(\mathrm{N}=43)\end{array}$ & $\mathrm{t}(d f)$ & p Value ${ }^{\mathrm{a}}$ \\
\hline Number of inpatient admissions & $3.00(2.33)$ & $0.93(.91)$ & $5.209(42)$ & $<0.0001$ \\
\hline Number of day-program admissions & $0.98(.89)$ & $0.63(.69)$ & $2.479(42)$ & 0.02 \\
\hline Number of inpatient and day-program admissions & $3.98(2.91)$ & $1.56(1.44)$ & $5.012(42)$ & $<0.0001$ \\
\hline Total treatment course (days) & $1038(585)$ & $540(441)$ & $4.647(42)$ & $<0.001$ \\
\hline
\end{tabular}

${ }^{\text {a} P a i r e d ~ S t u d e n t ' s ~} t$-test, two-tailed alpha level of 0.05 .

${ }^{\mathrm{b}}$ Drive for Thinness and Body Dissatisfaction are subscales of the Eating Disorder Inventory-2.

$\mathrm{BMI}=$ Body mass index $\mathrm{CDI}=$ Children's Depression Inventory; MASC $=$ Multidimensional Anxiety Scale for Children; $\mathrm{ED}=$ eating disorder. 
Table 4. Mean Medical and Psychological Variables of Interest at the Time of Initial Assessment, and Treatment Course Variables for a Sample of Inpatients Who Were Adjunctively Treated with Olanzapine Versus Those Who Were Not Adjunctively Treated with Olanzapine

\begin{tabular}{|c|c|c|c|c|}
\hline Baseline characteristics & $\begin{array}{c}\text { Olanzapine Mean }(S D) \\
(\mathrm{N}=11)\end{array}$ & $\begin{array}{l}\text { Comparison Mean (SD) } \\
\qquad(\mathrm{N}=11)\end{array}$ & $\mathrm{t}(d f)$ & $\mathrm{p}$ Value $^{\mathrm{a}}$ \\
\hline Age at admission (years) & $14.3(1.9)$ & $14.4(1.8)$ & $-0.971(10)$ & 0.355 \\
\hline Disease chronicity (months) & $10.36(8.04)$ & $9.73(5.62)$ & $0.194(10)$ & 0.850 \\
\hline BMI at admission $\left(\mathrm{kg} / \mathrm{m}^{2}\right)$ & $15.57(1.59)$ & $15.55(1.49)$ & $0.050(10)$ & 0.961 \\
\hline $\begin{array}{l}\text { Baseline psychological measures } \\
\quad \text { relating to comorbidity }\end{array}$ & $\begin{array}{l}\text { Olanzapine Mean (SD) } \\
\qquad(\mathrm{N}=7)\end{array}$ & $\begin{array}{l}\text { Comparison Mean (SD) } \\
\qquad(\mathrm{N}=7)\end{array}$ & $\mathrm{t}(d f)$ & p Value ${ }^{\mathrm{a}}$ \\
\hline CDI t-score & $81.43(16.58)$ & $53.43(15.60)$ & $2.949(6)$ & 0.026 \\
\hline MASC t-score & $63.29(11.21)$ & $54.00(7.59)$ & $1.441(6)$ & 0.200 \\
\hline $\begin{array}{l}\text { Baseline ED specific } \\
\quad \text { psychological measures }\end{array}$ & $\begin{array}{l}\text { Olanzapine Mean }(S D) \\
\quad(\mathrm{N}=6)\end{array}$ & $\begin{array}{l}\text { Comparison Mean }(S D) \\
\qquad(\mathrm{N}=6)\end{array}$ & $\mathrm{t}(d f)$ & p Value ${ }^{\mathrm{a}}$ \\
\hline Drive for Thinness ${ }^{\mathrm{b}}$ & $17.33(5.88)$ & $9.67(5.85)$ & $1.964(5)$ & 0.107 \\
\hline Body Dissatisfaction $^{\mathrm{b}}$ & $18.17(9.28)$ & $8.83(8.13)$ & $2.325(5)$ & 0.068 \\
\hline Treatment course & $\begin{array}{c}\text { Olanzapine Mean }(S D) \\
(\mathrm{N}=11)\end{array}$ & $\begin{array}{c}\text { Comparison Mean }(S D) \\
(\mathrm{N}=11)\end{array}$ & $\mathrm{t}(d f)$ & $\mathrm{p}$ Value $^{\mathrm{a}}$ \\
\hline Weight gain (kg) & $6.96(2.63)$ & $2.97(2.09)$ & $4.779(10)$ & 0.001 \\
\hline Length of stay (days) & $45.82(23.44)$ & $25.55(11.09)$ & $2.679(10)$ & 0.023 \\
\hline Rate of weight gain (kg/week) & $1.14(0.32)$ & $0.79(0.52)$ & $2.048(10)$ & 0.068 \\
\hline BMI at discharge $\left(\mathrm{kg} / \mathrm{m}^{2}\right)$ & $18.26(0.92)$ & $16.63(1.78)$ & $4.146(10)$ & 0.002 \\
\hline Day-program admissions & $1.00(0.77)$ & $0.91(0.94)$ & $0.289(10)$ & 0.779 \\
\hline Readmissions & $2.63(1.75)$ & $1.55(1.63)$ & $2.502(10)$ & 0.031 \\
\hline
\end{tabular}

${ }^{\text {a}}$ Paired Student's $t$-test, two-tailed alpha level of 0.05 .

${ }^{\mathrm{b}}$ Drive for Thinness and Body Dissatisfaction are subscales of the Eating Disorder Inventory-2.

$\mathrm{BMI}=$ body mass index $\mathrm{CDI}=$ Children's Depression Inventory; $\mathrm{ED}=$ eating disorder; MASC $=$ Multidimensional Anxiety Scale for Children.

\section{Ancillary testing}

Sixteen out of 43 patients treated with olanzapine (37\%) underwent a brain magnetic resonance imaging scan at some point during their treatment, compared with one $(2.3 \%)$ comparison participant $(p<0.001)$. The scans of two patients treated with olanzapine were reported as abnormal, although the findings were clinically insignificant.

\section{Course of treatment}

Almost all patients treated with olanzapine $(42 / 43,98 \%)$ were admitted on at least one occasion to intensive treatment programming (day hospital or inpatient), which was significantly higher than the comparison group $(32 / 43,74 \%, p=0.006)$. In fact, there were significant differences across multiple points relating to treatment focus and intensity between those treated with olanzapine and those not treated with the same (Table 3). Patients treated with olanzapine had higher overall numbers of inpatient admissions, day-treatment program admissions, and lengthier total treatment courses (i.e., time from initial assessment to last contact) (Table 3).

Data pertaining to the time of initiation of olanzapine, drug dosing, patient weight at drug initiation, and concurrent drug treatments have been presented in Table 5. As noted, patients started olanzapine at mean healthy body weights of $84 \%$ predicted and tended to be treated with relatively low doses (median dose $5 \mathrm{mg}$ ). Time at which olanzapine was initiated varied widelymore than three quarters of patients treated with olanzapine were started on the medication during an inpatient admission. Nearly half of the patients $(21 / 43)$ were receiving treatment with another medication at the time of olanzapine initiation, of which selective serotonin or norepinephrine reuptake inhibitors were the most common (Table 5). Three patients remained on olanzapine at the time of their transfer to adult care. The mean length of olanzapine treatment for the remaining 40 patients who discontinued the drug while under the care of our team was 252 days (SD 395.12 days).

Table 5. Treatment Variables Relating to the Initiation and Maintenance of Olanzapine Use

Treatment setting during olanzapine initiation $(N=43), N(\%)$

Inpatient admission 35 (81)

Partial hospitalization/day treatment 2 (5) program

Outpatient treatment

Weight variables at time of olanzapine

initiation $(N=42)$, Mean (SD)

Weight at olanzapine initiation $(\mathrm{kg}) \quad 42.6$ (7.9)

$\%$ mean healthy body weight at olanzapine $84(9.5)$ initiation

Concurrent medication use at olanzapine

initiation $(N=43), N(\%)$

SSRI/SNRI

Benzodiazepine

Olanzapine dosing $(N=43)$, Median (IQR) Maximum olanzapine dose used (mg)

$5.0(3.75$ to 7.5$)$

SSRI $=$ Selective serotonin reuptake inhibitor; SNRI = Selective norepinephrine reuptake inhibitor; $\mathrm{SD}=$ standard deviation; $\mathrm{IQR}=$ interquartile range. 
Table 6. Frequency and Percentage of Screening Tests Performed Before Olanzapine Initiation and During the Course of Olanzapine Treatment in a Sample of 43 Patients Treated With Olanzapine

\begin{tabular}{llc}
\hline Time of Screening & \multicolumn{1}{c}{ Screening test } & Frequency (\%) \\
\hline Before olanzapine initiation & Any screening test & $34(79)$ \\
& ECG & $31(72)$ \\
& HbA1c & $3(7)$ \\
& Fasting lipid profiles & $15(35)$ \\
& Fasting glucose & $17(40)$ \\
During olanzapine course & Fasting lipid profiles & $24(56)$ \\
\hline
\end{tabular}

$\mathrm{ECG}=$ electrocardiogram; HbA1c $=$ hemoglobin A1c.

\section{Screening tests}

Thirty-four patients (79\%) underwent some form of screening test before olanzapine initiation (Table 6). The most commonly completed screening test was the ECG, whereas hemoglobin A1c levels were least likely to be obtained. More than half of patients (24/43) had fasting lipid profiles completed at some point during their olanzapine course, although the specific timing of ongoing evaluation varied considerably between patients. Of the 31 patients who underwent initial ECG screening, repeat testing was completed in just 4 patients during their olanzapine course. Of these results, one patient experienced a borderline prolongation of the QT (QTc) interval while on olanzapine. The QTc corrected spontaneously on repeat testing 1 week later (while the patient remained on drugs) and was found to be borderline prolonged again 6 weeks after olanzapine termination, suggesting that the medication had not been directly responsible for the observed QTc change.

\section{Side-effect profile}

Twenty-four out of 43 patients (56\%) and/or treating physicians attributed at least one side effect to the medication, the most common of which was sedation (Table 7). Abnormalities in blood work followed as the second most common side effect; more than half of the patients in this subgroup $(4 / 7 ; 57 \%)$ stopped the drug because of these abnormalities. One patient was noted to have significantly elevated liver function tests 12 days after olanzapine initiation (ALT 457, AST 297, GGT 38), although liver function had been elevated at the time of her assessment 48 days earlier (ALT 114) and on recheck 23 days after assessment (ALT 101, AST 61). The most common abnormalities in blood work noted during laboratory monitoring were elevations in the fasting lipid profile. Seven of the 24 patients ( $29 \%$ [ $95 \%$ CI, $15 \%$ to $49 \%$ ]) who underwent fasting lipid profile testing experienced disturbances in lipid levels at some point during the medication trial. Many of the patients lacked completed fasting assessments before initiating olanzapine but in four instances, dyslipidemia occurred in the presence of documented baseline fasting levels that were within a normal range before initiating olanzapine (Table 7). Lipid screening was not available for patients in the matched sample and, thus, comparisons could not be made. Lipid abnormalities occurred on an average 10.4 weeks (SD 8.4) after drug initiation. No patient received targeted lipid therapy as a result of the observed abnormalities. Patient mean BMI at time of first detected lipid abnormality was 20.0 (SD 1.4), which corresponded to healthy body weight of $95 \%$ (SD 6).

The most commonly cited reasons noted in patients' charts for olanzapine termination were either related to improvements in patients' psychological status or weight restoration $(50 \%, 95 \% \mathrm{CI}$, $36 \%$ to $65 \%)$, patients' refusal to continue taking medication ( $20 \%$, $95 \% \mathrm{CI}, 11 \%$ to $34 \%)$, or undesirable side effects $(19 \%, 95 \% \mathrm{CI}$, $10 \%$ to $33 \%$ ). Interestingly, $36 \%$ of patients ( $95 \%$ CI, $23 \%$ to $50 \%$ ) restarted the olanzapine after initial discontinuation, most commonly because of increased psychological distress or pronounced weight loss.

\section{Discussion}

This is the largest review to date describing olanzapine use for the adjunctive treatment of EDs, and the most comprehensive review available addressing olanzapine's tolerability in an adolescent population with AN or EDNOS. Treatment with olanzapine was notable over the study period. This study suggests that patients treated with olanzapine were more likely to present with greater degrees of underlying psychopathology than those not treated with olanzapine, as evidenced by significantly lower BMIs at assessment, higher rates of comorbid anxiety and depression, greater rates of admission to intensive treatment, subsequent readmission, greater length of stays, and total treatment course.

The observed rates of comorbid anxiety disorders and depression in this study are in keeping with previously published reports,

Table 7. Frequency and Percentage of Side Effects Endorsed by 43 Participants or Reported by the Participants' Treating Physician During the Course of Olanzapine Treatment

\begin{tabular}{|c|c|c|}
\hline & Frequency (\%) & $\begin{array}{c}\text { Prelpost treatment } \\
\text { lipid values }(\mathrm{mmol} / \mathrm{L})\end{array}$ \\
\hline \multicolumn{3}{|l|}{ Side effect } \\
\hline Any side effect $(N=43)$ & $24(56)$ & \\
\hline Sedation $(N=43)$ & $17(40)$ & \\
\hline Lipid abnormality (24 patients screened) & $7(29)$ & \\
\hline \multicolumn{3}{|c|}{$\begin{array}{l}\text { Patients with documented normal baseline lipid values } \\
\text { who experienced disturbances over the course } \\
\text { of the medication trial }\end{array}$} \\
\hline Increased cholesterol (24 patients screened) & & $5.8 \rightarrow 6.5$ \\
\hline Normal reference range $(3.2-5.8 \mathrm{mmol} / \mathrm{L})$ & $3(13)$ & $\begin{array}{l}5.3 \rightarrow 6.6 \\
3.5 \rightarrow 5.5\end{array}$ \\
\hline $\begin{array}{l}\text { Increased LDL ( } 24 \text { patients screened) } \\
\text { Normal reference range }(1.5-3.6 \mathrm{mmol} / \mathrm{L})\end{array}$ & $1(4)$ & $2.95 \rightarrow 5.05$ \\
\hline
\end{tabular}

$\mathrm{LDL}=$ low density lipoprotein. 
although reported prevalence across AN populations have varied widely, with lifetime prevalence rates of at least one anxiety disorder ranging from 23\% to 75\% (Godart et al. 2002; Swinbourne and Touyz 2007) and depression from $10 \%$ to $71 \%$ (Godart et al. 2007). Concurrent use of other psychotropic medication was extremely high in our sample, with almost all concurrent pharmacotherapy targeting comorbid anxiety or depressive symptoms.

The consistency of psychological measures completed at assessment varied over the study period. Baseline psychological testing of patients began in 2001 in our center; therefore, a percentage of patients within this study timeframe went without this component of assessment. Despite this, mean scores in all analyzed measures showed greater elevation in the olanzapine-treated cohort. Clinically, this lends support to the hypothesis that patients augmented with olanzapine presented with greater degrees of psychopathology at assessment, although a larger sample would be necessary to delineate any true differences. Given the high levels of defensiveness in this population, and the egosyntonic nature of the illness, baseline scores should be interpreted with caution.

Analysis of weight gain using the inpatient cohort proved interesting from multiple standpoints. First, unlike the overall cohort of patients analyzed, there was no observed difference in BMIs at assessment in this group. Second, observed differences in rates of comorbidity were less, although significantly higher rates of depression were still observed. Despite this, length of stay, and rates of readmission were greater in those subsequently augmented with olanzapine. Thus, despite our inability to differentiate these patients as well (in terms of measurable differences between groups), treatment courses in those augmented with olanzapine again suggested greater illness severity, suggesting other extenuating factors that have not been accounted for in this review. Weight gain was only examined in the inpatient sub-cohort in order to minimize as many confounders as possible. Many of these confounders would have been based in the retrospective nature of the study and our inability to control for multiple potential mitigating factors (including mode of treatment being offered at olanzapine initiation, timing of pharmacotherapy initiation in relation to course of treatment, consistency of dosing schedules, nutritional prescription and intake, intensity of activity regimens, etc). Based on this analysis, there was no significant difference in the mean rate of weekly weight gain although it is possible that with a greater sample size, this could be shown otherwise. Given the underlying differences between groups, it is difficult, however, to delineate any true conclusions.

Although our standard practice is not to complete brain magnetic resonance imaging evaluations in patients with $\mathrm{AN}$, a large percentage of patients who received olanzapine did undergo such testing, suggesting some atypicality of their presentation or disease course. Again, this finding is in keeping with the working hypothesis that patients treated with olanzapine were, in fact, more ill.

The most common dose of olanzapine used in our sample was $5 \mathrm{mg}$ daily, although doses as high as 10 to $12.5 \mathrm{mg} /$ day have been reported elsewhere (Mehler-Wex et al. 2008). Although the body of evidence investigating the potential role for adjunctive olanzapine therapy in severely ill individuals with AN is growing, data regarding tolerability are sparse. Studies completed in non-ED adolescent populations have demonstrated substantial concerns with excessive weight gain, dyslipidemia, and metabolic disturbances in populations treated with atypical antipsychotics, especially olanzapine (Arango et al. 2009; Kryzhanovskaya et al. 2009; Swadi et al. 2010). Our study suggests a high-observed rate of side effects attributable to olanzapine, with the most commonly cited side ef- fect being sedation. Bissada et al. (2008) noted no serious adverse effects in their study. In contrast, over half of our patients experienced at least one side effect attributed to olanzapine (albeit almost all of these side effects would not be classified as serious), but more concerning from a clinical standpoint, almost $20 \%$ of patients discontinued treatment as a direct result, underlying the need for close monitoring (Bissada et al. 2008). Although not specifically coded in this study, patient fears concerning excessive weight gain (whether real or perceived) influenced initiation rates as well as rates and timing of drug discontinuation. Concerns associated with medication effects and refusal to consider medication as a treatment option have been cited as the most common reasons for patients to refuse participation in randomized controlled studies investigating olanzapine use as augmented treatment for AN (Norris et al. 2010). Although many published articles contain generic statements regarding tolerability, the degree to which patients are screened medically while on the medication is often not reported or is unclear. Our personal experience has evolved over the last decade, owing, in part, to side effects we have encountered.

The rate at which patients were screened at the time of olanzapine initiation varied significantly depending on the screening test. Ongoing evaluation of serum glucose, lipid levels, and ECGs while on treatment were even more inconsistent. Although almost three quarters of patients received baseline ECGs, less than half received screening lipid or fasting glucose profiles. The very low rates of repeat ECG assessment while on olanzapine is also of concern, given the independent risks of QTc prolongation associated with olanzapine use and AN (Cooke et al. 1994; Titier et al. 2005; Ritchie and Norris 2009). Further exploration into what factors may increase risk in this area would help guide clinicians' monitoring practice.

A recent study examining rates of lipid and glucose screening among adult patients initiating second generation atypical antipsychotics (SGA) found that on average $<20 \%$ of individuals initiating SGA drug therapy received baseline glucose testing and $<10 \%$ received baseline lipid testing (Titier et al. 2005). This is relevant as psychotropic medications, including olanzapine, have been linked to adiposity-dependent and potentially adiposityindependent changes in insulin sensitivity and lipid metabolism (Newcomer 2007). Olanzapine use appears to confer an increased risk of dyslipidemia and DM compared with other atypical agents (Newcomer 2007). To date, there have been no case reports of olanzapine-induced DM in patients with AN, although olanzapine's effect on glucose, independent of adiposity, has been demonstrated in some patients with schizophrenia in a small clinical trial (Newcomer et al. 2002). Olanzapine's effect on lipid metabolism, however, has almost always been associated with weight gain in treated patients. A recent case report of a patient with schizophrenia treated with olanzapine did demonstrate elevated serum triglyceride levels despite the absence of weight gain and abnormal glucose metabolism (Nagamine 2008). Although our sample size is small, the present study would support the likelihood that the mechanism of action contributing to dyslipidemia is not solely dependent on a patient's absolute weight, as a few of our patients developed lipid profile abnormalities at weights that were either below or approaching healthy individualized ranges. Although we do not have comparative lipid data on the cohort of patients not treated with olanzapine, lipid disturbances that were noted in treated patients evolved after starting olanzapine. In four of these cases, baseline blood work had demonstrated normal total cholesterol and LP values before initiating treatment. Although 
multiple studies in low-weight patients with AN have demonstrated high serum levels of total cholesterol and LP before initiating refeeding, studies that have reported on the evolution of lipid profiles during refeeding have generally shown that lipid levels either improve or remain stable (Rigaud et al. 2009; Arden et al. 1990; Weinbrenner et al. 2004). To the best of our knowledge, no study has demonstrated that lipid values get worse with standard refeeding (especially after baseline data have been collected and shown to be normal). Considering the fact that olanzapine's effect on dyslipidemia has been well established, the authors conclude that the observed dyslipidemia appears at least in a percentage of patients to be attributable to the medication use.

Comments regarding the potential efficacy of olanzapine using this data set cannot be made, as it is clear that both groups of patients are, in fact, different. An attempt was made to match patients on diagnosis, age, and treatment modality; however, the significant differences in comorbidity undoubtedly affected the overall clinical course of patients treated with olanzapine. With that said, attempting to match patients based on diagnosed comorbidity would prove challenging, although it would possibly lend greater clues into answering the question of whether augmenting patients with olanzapine aids treatment. In hindsight, the results of this study are not completely surprising, given the fact that during the treatment time frame it was common practice to prescribe olanzapine in "treatment-resistant" cases. Interestingly, one third of observed patients who stopped taking olanzapine prematurely/on their own re-initiated the drug after experiencing either increased psychological distress or pronounced weight loss post-discontinuation. This could suggest some potential benefit to being on the medication (whether real or perceived), although a causal inference cannot be made.

Specific limitations of this study include its retrospective nature (and its impact on accumulation of a complete data set) and a relatively small sample size. A number of potential confounding factors have also been discussed above.

\section{Conclusions}

Overall, the results from this study provide some insight into the clinical parameters that may be driving olanzapine prescription as an adjunctive treatment for youth with AN and EDNOS. The cohort of patients who received treatment with olanzapine in our center presented with greater acuity and more complex psychopathology than those patients not treated with olanzapine. Based on available data, the observed side-effect profile of patients treated with olanzapine indicates the need for close monitoring during the entire course of treatment. Although the data are limited by a small power, the rate of weight gain while on olanzapine in this sample was shown to be greater in patients treated with olanzapine, despite other indicators of greater illness severity. It is possible that with a larger sample, this result could attain statistical significance. The absence of glucose-related abnormalities while on olanzapine is reassuring, although the observed lipid abnormalities affirm the need for close metabolic monitoring when treatment is prolonged and the establishment of regular monitoring practices in further prospective studies.

There are many unanswered questions regarding olanzapine use in this population. Although this article suggests that factors which may lead physicians to prescribe adjunctive therapy in this cohort may be linked to acuity and associated comorbidity, comparison of cohorts matched for comorbidity could lend greater information regarding efficacy. Large-scale studies examining patient charac- teristics that may be receptive to olanzapine use could also help clinicians in deciding which cohort of patients may be most suited for adjunctive olanzapine therapy. Prospective randomized placebocontrolled studies with adequate power are required to further assess the safety profile of such medications in this population and, more specifically, to further investigate whether olanzapine does, in fact, confer some advantage in treatment.

\section{Clinical Significance}

Overall, the results from this study provide some insight into the clinical parameters that may be driving olanzapine prescription as an adjunctive treatment for youth with AN and EDNOS. Patients treated with olanzapine presented with greater acuity and more complex psychopathology than those patients not treated with olanzapine, which made comparisons regarding efficacy of the drug impossible. The observed side-effect profile noted in patients treated with olanzapine indicates the need for close monitoring during the entire course of treatment, regardless of the patient's absolute weight.

\section{Disclosures}

Mark L. Norris, M.D., Wendy Spettigue, M.D., Annick Buchholz, Ph.D., Katherine A. Henderson, Ph.D., Rebecca Gomez, B.Sc., Danijela Maras, B.Sc., Isabelle Gaboury, Ph.D., and Andy Ni, M.Sc.: No financial relationships with any pharmaceutical company.

\section{References}

American Psychiatric Association. Diagnostic and Statistical Manual of Mental Disorders, 4th ed. (DSM-IV). Washington, DC: American Psychiatric Association; 1994.

American Psychiatric Association. Treatment of Patients with Eating Disorders. American Psychiatry Publishing, Inc., 2006; Available at: www.psychiatryonline.com/pracGuide/pracGuideTopic_12.aspx. Accessed May 21, 2008.

Arango C, Robles O, Parellada M, Fraguas D, Ruiz-Sancho A, Medina O, Zabala A, Bombín I, Moreno D: Olanzapine compared to quetiapine in adolescents with a first psychotic episode. Eur Child Adolesc Psychiatry 18:418-428, 2009.

Arden MR, Weiselberg EC, Nussbaum MP, Shenker IR, Jacobson MS: Effect of weight restoration on the dyslipoproteinaemia of anorexia nervosa. J Adolesc Health Care 11:199-202, 1990.

Barbarich NC, McConaha CW, Gaskill J, La Via M, Frank GK, Achenbach S, Plotnicov KH, Kaye W: An open trial of olanzapine in anorexia nervosa. J Clin Psychiatry 65:1480-1482, 2004.

Bissada H, Tasca GA, Barber AM, Bradwejn J: Olanzapine in the treatment of low body weight and obsessive thinking in women with anorexia nervosa: A randomized, double-blind, placebo-controlled trial. Am J Psychiatry 165:1281-1288, 2008.

Boachie A, Goldfield GS, Spettigue W: Olanzapine use as an adjunctive treatment for hospitalized children with anorexia nervosa: Case reports. Int J Eat Disord 33:98-103, 2003.

Brambilla F, Garcia CS, Fassino S, Daga GA, Favaro A, Santonastaso P, Ramaciotti C, Bondi E, Mellado C, Borriello R, Monteleone P: Olanzapine therapy in anorexia nervosa: Psychobiological effects. Int Clin Psychopharmacol 22:197-204, 2007.

Citrome L: Olanzapine: Interpreting the label change. Int J Clin Pract 61:1960-1962, 2007.

Cooke RA, Chambers JB, Singh R, Todd GJ, Smeeton NC, Treasure J, Treasure T: QT interval in anorexia nervosa. Br Heart J 72:69-73, 1994. 
Eli Lilly and Company. Zyprexa (olanzapine) tablets [product information handout]. Indianapolis, Ind. 2008; Available at: http:// pi.lilly.com/us/zyprexa-pi.pdf. Accessed April 24, 2008.

Garner DM: EDI-2: Eating Disorder Inventory-2 Professional Manual. Odessa, Florida: Psychological Assessment Resources, Inc.; 1991.

Garner DM, Garfinkel PE: Handbook of Treatment for Eating Disorders, 2nd ed. New York: The Guilford Press; 1997.

Godart NT, Flament MF, Perdereau F, Jeammet P: Comorbidity between eating disorders and anxiety disorders: A review. Int $\mathrm{J}$ Eat Disord 32:253-270, 2002.

Godart NT, Perdereau F, Rein Z, Berthoz S, Wallier J, Jeammet P, Flament MF: Comorbidity studies of eating disorders and mood disorders. Critical review of the literature. J Affect Disord 97:3749, 2007.

Kovacs M: The Child Depression Inventory (CDI). Psychopharmacol Bull 21:995-998, 1981.

Kryzhanovskaya L, Schulz SC, McDougle C, Frazier J, Dittmann R, Robertson-Plouch C, Bauer T, Xu W, Wang W, Carlson J, Tohen M: Olanzapine versus placebo in adolescents with schizophrenia: A 6-week, randomized, double-blind, placebo-controlled trial. J Am Acad Child Adolesc Psychiatry 48:60-70, 2009.

Lucas AR, Beard CM, O'Fallon WM, Kurland LT: 50-year trends in the incidence of anorexia nervosa in Rochester, Minn.: A populationbased study. Am J Psychiatry 148:917-922, 1991.

March JS: Multidimenstional Anxiety Scale for Children Technical Manual. Toronto, Canada: Multi-Health Systems, Inc.; 1997.

Mehler-Wex C, Romanos M, Kirchheiner J, Schulze UM: Atypical antipsychotics in severe anorexia nervosa in children and adolescentsreview and case reports. Eur Eat Disord Rev 16:100-108, 2008.

Mondraty N, Birmingham CL, Touyz S, Sundakov V, Chapman L, Beumont P: Randomized controlled trial of olanzapine in the treatment of cognitions in anorexia nervosa. Australas Psychiatry 13:72-75, 2005.

Nagamine T: Olanzapine-induced elevation of serum triglyceride levels in a normal weight patient with schizophrenia. Intern Med 47:181-182, 2008.

Newcombe RG: Two-sided confidence intervals for the single proportion: Comparison of seven methods. Stat Med 17:857-872, 1998.

Newcomer JW: Antipsychotic medications: Metabolic and cardiovascular risk. J Clin Psychiatry 68:8-13, 2007.

Newcomer JW, Haupt DW, Fucetola R, Melson AK, Schweiger JA, Cooper BP, Selke G: Abnormalities in glucose regulation during antipsychotic treatment of schizophrenia. Arch Gen Psychiatry 59:337-345, 2002.

Norris ML, Spettigue W, Buchholz A, Henderson KA, Obeid N. Factors influencing research drug trials in adolescents with anorexia nervosa. Eat Disord 18:210-217, 2010.

Rigaud D, Tallonneau I, Vergès B: Hypercholesterolaemia in anorexia nervosa: Frequency and changes during refeeding. Diabetes Metab 35:57-63, 2009.

Ritchie B, Norris M: QTc prolongation associated with atypical antipsychotic use in the treatment of adolescent-onset anorexia nervosa. J Can Acad Adolesc Psychiatry 18:60-63, 2009.

Rivas-Vazquez RA, Rice J, Kalman D: Pharmacotherapy of obesity and eating disorders. Prof Psychol Res Pract 34:562-566, 2003.

Swadi HS, Craig BJ, Pirwani NZ, Black VC, Buchan JC, Bobier CM: A trial of quetiapine compared with risperidone in the treatment of first onset psychosis among 15- to 18-year-old adolescents. Int Clin Psychopharmacol 25:1-6, 2010.

Swinbourne JM, Touyz SW: The co-morbidity of eating disorders and anxiety disorders: A review. Eur Eat Disord Rev 15:253-274, 2007.

Titier K, Girodet PO, Verdoux H, Molimard M, Begaud B, Haverkamp W, Lader M, Moore N: Atypical antipsychotics: From potassium channels to torsade de pointes and sudden death. Drug Saf 28:35-51, 2005.

Tschoner A, Engl J, Laimer M, Kaser S, Rettenbacher M, Fleischhacker WW, Patsch JR, Ebenbichler CF: Metabolic side effects of antipsychotic medication. Int J Clin Pract 61:1356-1370, 2007.

Weinbrenner T, Züger M, Jacoby GE, Herpertz S, Liedtke R, Sudhop T, Gouni-Berthold I, Axelson M, Berthold HK: Lipoprotein metabolism in patients with anorexia nervosa: A case-control study investigating the mechanisms leading to hypercholesterolaemia. $\mathrm{Br}$ J Nutr 91:959-69, 2004.

\section{Address correspondence to: \\ Mark Norris, M.D., FRCPC \\ Paediatrics and Adolescent Medicine \\ Department of Paediatrics \\ Children's Hospital of Eastern Ontario \\ 401 Smyth Road \\ Ottawa, ON \\ Canada K1H $8 L 1$}

E-mail: mnorris@cheo.on.ca 